\title{
Low Energy Tandem Mass Spectrometry of Deoxynucleoside Adducts of Polycyclic Aromatic Hydrocarbon Dihydrodiol-Epoxides
}

\author{
Paula S. Branco,* M. Paul Chiarelli, and Jackson O. Lay, Jr. \\ National Center for Toxicological Research, Jefferson, Arkansas, USA and University of Arkansas-Little Rock, \\ Little Rock, Arkansas, USA
}

\author{
Frederick A. Beland \\ National Center for Toxicological Research, Jefferson, Arkansas, USA
}

\begin{abstract}
The use of fast-atom bombardment ionization-tandem mass spectrometry approaches, with collision energies on the order of $30-50 \mathrm{eV}$, was developed for the analysis of low picomole quantities of polycyclic aromatic hydrocarbon dihydrodiol-epoxide deoxynucleoside adducts. This strategy combines three experimental techniques: (1) product ion scans, (2) constant neutral loss scans, and (3) precursor ion scans. Product ion scans of the protonated molecule or the $\mathrm{BH}_{2}^{+}$ion that results from loss of the deoxyribose were dominated by fragments associated with cleavage of the sigma bond between the dihydrodiol-epoxide moiety and the nucleobase. Constant neutral loss scans were based upon the loss of deoxyribose (116 u) or the combined loss of the deoxynucleoside, water, and carbon monoxide ( $313 \mathrm{u}$ ); precursor ion scans utilized the latter fragment. The formation of trimethylsilyl derivatives increased the sensitivity of analysis, which allowed the simultaneous detection of DNA adducts in a mixture. (J Am Soc Mass Spectrom 1995, 6, 248-256)
\end{abstract}

$\mathrm{P}$ olycyclic aromatic hydrocarbons (PAHs) are a group of structurally related chemicals that constitute an important class of carcinogenic environmental pollutants [1]. These compounds, which are produced during combustion processes, are metabolized into electrophilic derivatives, typically dihydrodiol-epoxides, that are capable of reaction with DNA to elicit mutagenic and carcinogenic responses [2]. The analysis of carcinogen-DNA adducts that result from $\mathrm{PAH}$ dihydrodiol-epoxides or electrophilic derivatives from other classes of carcinogens has been proposed as a means of evaluation of the extent of human exposure to specific carcinogens [3]. DNA adduct analyses are complicated, however, by the low frequency of DNA adduction that occurs in vivo. In experimental animals, for example, only about $10-100$ pmol of adduct per milligram of DNA are normally formed following treatment with tumorigenic doses of carcinogens. This problem is further complicated by the fact that DNA is a minor component (about $0.1 \%$ by weight) of tissue. In addition, humans usually are exposed to smaller amounts of carcinogens than those used with experi-

Address reprint requests to Dr. Jackson O. Lay, Jr., Division of Chemistry, National Center for Toxicological Research, HFT 230, Jefferson, AR 72079

* Permanent address: Secçāo de Química Aplicada, Departamento de Quimica, Campus FCT-UNL Quinta da Torre, 2825 Monte da Caparica, Portugal mental animals, with resultant lower levels of DNA adduction [4].

Several techniques, which include immunoassays [5], fluorescence assays [6], and ${ }^{32}$ P-postlabeling [7], have been developed for the analysis of small quantities of carcinogen-DNA adducts. Although they are highly sensitive, these methods do not permit structure elucidation, which is a severe limitation when there is uncertainty with regard to the nature of the carcinogen exposure. Mass spectrometry has the potential to provide structural information and, as such, has played an important role in the structural elucidation of covalently modified nucleic acids [8].

High resolution electron impact mass spectrometry (EI-MS) has been used to determine the structures of several PAH dihydrodiol-epoxide DNA adducts [9]. However, the extreme polarity and thermal lability of these adducts necessitated the formation of methyl or silyl ethers prior to analysis via electron impact (EI) ionization. Because of the combined sensitivity losses associated with the use of high resolution and TMS derivatization, alternatives to the high resolution EI-MS method for measurement of adduct levels in human DNA warrant investigation, particularly for analyses that involve unknown carcinogen-DNA adducts.

Significantly less work has been reported on the tandem mass spectrometry of PAH dihydrodiolepoxide DNA adducts. A deoxyguanosine adduct of 
anti-benzo[ $a]$ pyrene-7,8-dihyd rodiol-9,10-epoxide (anti-BaPDE) was characterized by tandem mass spectrometry methods that used field desorption (FD) ionization and collision-induced dissociation (CID) under high collision energy ( $8 \mathrm{keV}$ ) conditions [10,11]. The fragment ions generated in the presence of the helium collision gas were readily interpretable in terms of the assigned structure and were similar to fragment ions observed in EI mass spectra of the permethylated adduct [9]. Fast-atom bombardment (FAB) product-ion mass spectrometry studies of BaPDE adducts recently have been conducted via a VG Analytical (Fisons, Danvers, MA) ZAB-T four-sector (BEBE) mass spectrometer [12]. The product ion spectra for these adducts were dominated by ions derived from the carcinogen moiety. Other tandem mass spectrometry analyses of dihydrodiol-epoxide adducts have been limited primarily to studies that concern the site of carcinogen attachment to the nucleobase [13, 14]. A relatively limited number of tandem mass spectrometry studies have been conducted with derivatized PAH-DNA adducts; however, trimethylsilyl (TMS) derivatives of deoxynucleoside-carcinogen adducts showed surprising sensitivity by fast-atom bombardment-mass spectrometry (FAB-MS), to an extent that appeared to be greater than EI-MS $[15,16]$.

In this work, we report the development of FAB tandem mass spectrometry methods for the analysis of PAH dihydrodiol-epoxide deoxynucleoside adducts. This work, which is an extension of our previous studies with arylamine-DNA adducts [17], combines three experimental approaches: (1) constant neutral loss scans, (2) product ion scans of the protonated molecule or the $\mathrm{BH}_{2}^{+}$ion that results from loss of deoxyribose, and (3) precursor ion scans. We show that the sensitivity for analysis is increased by formation of TMS derivatives, which allows detection of PAH dihydrodiol-epoxide deoxynucleoside adducts at low picomole levels. In addition, we demonstrate that FAB tandem mass spectrometry, especially of derivatized adducts, provides a rapid, sensitive, and selective method for the analysis of complex mixtures, often with minimal, if any, sample cleanup.

\section{Experimental}

\section{Materials}

The following PAH dihydrodiol-epoxides were purchased from the NCI Chemical Carcinogen Reference Standard Repository (Midwest Research Institute, Kansas City, MO): anti-benzo[ $k$ ]fluoranthene-trans8,9-dihydrodiol-10,11-epoxide (anti-benzo[ $k]$ fluoranthene dihydrodiol-epoxide), anti-benzo[ $b]$ fluoranthene-trans-9,10-dihydrodiol-11,12-epoxide (antibenzo[ $b]$ fluoranthene dihydrodiol-epoxide), anti-chrysene-trans-1,2-dihydrodiol-3,4-epoxide (anti-chrysene dihydrodiol-epoxide), anti-benz $(a]$ anthracene-trans3,4-dihydrodiol-1,2-epoxide (anti-benz[ $a$ ]anthracene dihydrodiol-epoxide), anti-dibenz $[a, c]$ anthracenetrans-10,11-dihyd rodiol-12,13-epoxide (antidibenz $[a, c]$ anthracene dihydrodiol-epoxide), and antiBaPDE. N-Trimethylsilylimidazole in pyridine (TMSI; Tri-Sil " $Z$ ") was acquired from Pierce Chemicals (Rockford, IL). 7,8,9,10-Tetrahyd roxy-7,8,9,10tetrahydrobenzo[ $a]$ pyrene was prepared by treating anti-BaPDE with dilute hydrochloric acid for $18 \mathrm{~h}$ at $37^{\circ} \mathrm{C}$.

\section{Adduct Synthesis and Derivatization}

Covalent modification of DNA by dihydrodiol-epoxides of PAIIs. To $1.2 \mathrm{mg}$ of salmon testes DNA (Sigma Chemical Co., St. Louis, MO) in $1 \mathrm{~mL}$ of $5-\mathrm{mM}$ BisTris-HCl (Sigma), 0.1-mM EDTA, pH 7.1, was added $159 \mu \mathrm{g}$ of anti-BaPDE in $107 \mu \mathrm{L}$ of tetrahydrofuran. The mixture was allowed to react at $37^{\circ} \mathrm{C}$ for $24 \mathrm{~h}$ and then was extracted with $1 \mathrm{~mL}$ of redistilled phenol saturated with an equal volume of $50-\mathrm{mM}$ Bis-Tris- $\mathrm{HCl}$, 1-mM EDTA, pH 7.1. Additional extractions were conducted with a mixture of $500 \mu \mathrm{L}$ of phenol and $500 \mu \mathrm{L}$ of Sevag (chloroform:isoamyl alcohol, 24:1), and then 1 $\mathrm{mL}$ of Sevag. To the aqueous phase was added $100 \mu \mathrm{L}$ of $5-\mathrm{M} \mathrm{NaCl}$ and $1.1 \mathrm{~mL}$ of cold ethanol. The precipitated DNA was collected by centrifugation, washed twice with $70 \%$ ethanol, and added to $1 \mathrm{~mL}$ of $5-\mathrm{mM}$ Bis-Tris- $\mathrm{HCl}, 0.1-\mathrm{mM}$ EDTA, pH 7.1. The level of modification, as determined by UV spectrometry, with an $\epsilon_{347}=29,000 \mathrm{mM}^{-1}$ [18], was $1.96 \%$. The modified DNA was hydrolyzed as previously described [19] and the adducts were purified by high performance liquid chromatography (HPLC) with a $3.9-\times 300-\mathrm{mm}$ $\mu$ Bondapak $\mathrm{C}_{18}$ column (Waters Associates, Milford, $\mathrm{MA}$ ) and a 30-min linear gradient of 20-100\% methanol at a flow of $2 \mathrm{~mL} / \mathrm{min}$. A $500-\mathrm{MHz}{ }^{1} \mathrm{H}-\mathrm{NMR}$ spectrum of the major adduct was obtained and it was consistent with $N^{2}$-deoxyguanosine substitution at C10 of antiBaPDE. Similar procedures were used for reactions with the other PAH dihydrodiol-epoxides. The levels of modification from these dihydrodiol-epoxides were estimated by UV spectrometry by using the molar absorptivity value for the dihydrodiol-epoxides provided by the supplier.

Reaction between nucleosides with anti-BaPDE. To 10 $\mathrm{mg}$ of deoxyguanosine (U.S. Biochemical, Cleveland, $\mathrm{OH} ; 35 \mu \mathrm{mol}$ ) in $500 \mu \mathrm{L}$ of dimethylsulfoxide (DMSO) was added $60 \mu \mathrm{g}$ of anti-BaPDE $(0.2 \mu \mathrm{mol})$ and $10 \mu \mathrm{L}$ of triethylamine. After $24 \mathrm{~h}$ at $37{ }^{\circ} \mathrm{C}$, the reaction mixture was evaporated, $1 \mathrm{~mL}$ of water was added, and the mixture was extracted three times with diethyl ether to remove residual anti-BaPDE and degradation products. The aqueous phase was then applied to a C-18 Sep-Pak (Waters Associates), eluted with water to remove unreacted deoxyguanosine, and then eluted with methanol to recover the deoxyguanosine adduct in $18 \%$ yield. The adduct was further purified by HPLC, with a $\mu$ Bondapak column and the solvent 
conditions described previously. The same procedure was used with deoxyadenosine and deoxycytidine. ${ }^{1} \mathrm{H}$ NMR spectra were obtained for the deoxyadenosine and deoxycytidine adducts and both were consistent with exocyclic amine substitution at $\mathrm{C} 10$ of anti-BaPDE.

Reaction between nucleoside bases and anti-BaPDE. To $12.7 \mathrm{mg}$ of guanine (Sigma; $84 \mu \mathrm{mol}$ ) in $1 \mathrm{~mL}$ of DMSO was added $200 \mu \mathrm{g}$ of anti-BaPDE and $30 \mu \mathrm{L}$ of triethylamine. Because the guanine was insoluble, the reaction was heated to $60{ }^{\circ} \mathrm{C}$. HPLC analysis after $24 \mathrm{~h}$ indicated the formation of a product in very low yield. Due to the low yield, the adduct was not isolated and FAB tandem mass spectrometry was performed directly on the reaction mixture. The same procedure was used with adenine and cytosine, except that the reactions were conducted at $37^{\circ} \mathrm{C}$ for $24 \mathrm{~h}$.

Derivatization procedures. Adducts were silylated by adding $10 \mu \mathrm{L}$ of TMSI to $1 \mu \mathrm{g}$ of dry sample and heating at $65^{\circ} \mathrm{C}$ for $2 \mathrm{~h}$. The sample was then dried in vacuo and diluted with DMSO prior to analysis. When modified DNA was analyzed, $100 \mu \mathrm{L}$ of TMSI was added to the hydrolyzed samples.

\section{Mass Spectrometry}

After application of 1-2 $\mu \mathrm{L}$ of thioglycerol FAB matrix liquid, $0.5-1.0 \mu \mathrm{L}$ of a solution that contained the sample was applied directly to a copper target with a syringe. Spectra were obtained via a Finnigan-MAT (San Jose, CA) TSQ-70 with an Ion Tech (Middlesex, UK) gun. FAB ionization was effected by using xenon atoms accelerated to $8-10 \mathrm{keV}$. Scans were obtained from approximately $50-700 \mathrm{u}$ under control of the TSQ-70 data system. Constant neutral loss scans were acquired by scanning the first and last quadrupoles (Q1 and Q3) at the same rate, with a constant mass offset that corresponded to either the mass of the deoxyribose moiety (116 u) or the loss of $313 \mathrm{u}$. Product ion scans were obtained by fixing the mass passed by $\mathrm{Q} 1$ to correspond to the protonated molecule or the $\mathrm{BH}_{2}^{+}$ion, whereas $\mathrm{Q} 3$ was scanned to identify the masses of all the fragments formed by decomposition of the selected precursor ion. Precursor ion scans were obtained by selecting, with Q3, a specific product ion, whereas the first analyzer (Q1) was scanned to select all precursor ions of that fragment. All scans were acquired by using argon as the collision gas. Collision energies of $30-50 \mathrm{eV}$ were used and collision cell pressures were varied to maximize the yield of useful product ions.

\section{Results and Discussion}

We previously demonstrated that chemical derivatization coupled with FAB-MS is a sensitive method for the analysis of arylamine-DNA adducts [17]. In a continuation of this work, we now have determined that FAB-MS is a sensitive technique for the detection of DNA adducts derived from PAH dihydrodiolepoxides. We also have established the types of structural information that can be obtained from FAB tandem mass spectrometry on these compounds.

\section{Fast-Atom Bombardment Mass Spectrometry}

Compared to the arylamine-deoxynucleoside adducts we studied previously [17], the PAH-deoxynucleoside adducts gave very poor $F A B$ full scan mass spectra. Figure 1a shows a FAB mass spectrum from $100 \mathrm{ng}$ of the deoxyguanosine adduct obtained from anti-chrysene dihydrodiol-epoxide. The ion of $\mathrm{m} / \mathrm{z} 546$ corresponds to the protonated molecule and that at $\mathrm{m} / \mathrm{z}$ 430 to the $\mathrm{BH}_{2}^{+}$ion that results from loss of the deoxyribose moiety. Better results were obtained when the adducts were derivatized with TMSI; for example, the high mass region of a FAB mass spectrum for $5 \mathrm{ng}$ of the anti-chrysene dihydrodiol-epoxide deoxyguanosine adduct after TMSI derivatization is shown in Figure 1b. The ions at $m / z 646$ and $m / z 762$ correspond, respectively, to tris-TMS derivatives of the $\mathrm{BH}_{2}^{+}$ ion and the protonated molecule. The observation of a tris-TMS-modified $\mathrm{BH}_{2}^{+}$ion $(m / z$ 646) $116 \mathrm{u}$ lower in mass than the tris-TMS-derivatized protonated molecule indicates that for at least some of the tris-TMS-modified protonated molecules, all of the

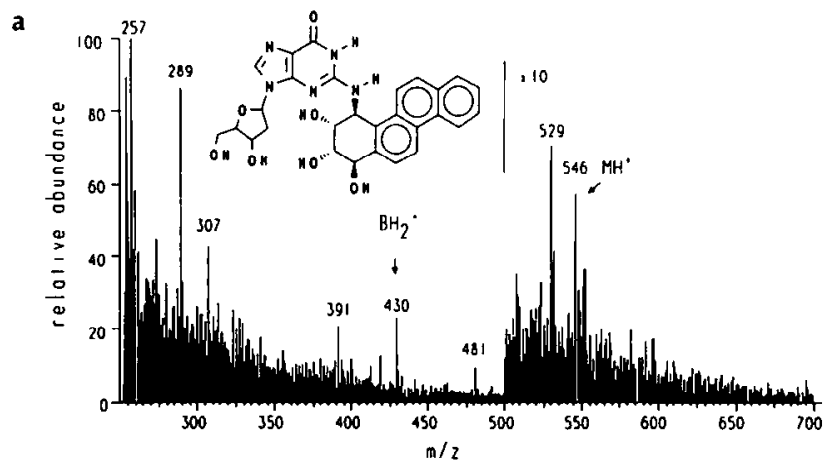

b

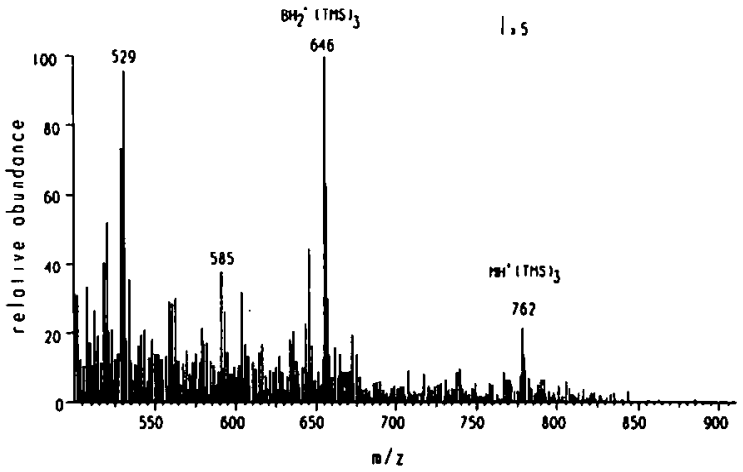

Figure 1. FAB mass spectra of anti-chrysene dihydrodiolepoxide deoxyguanosine adduct (a) before and (b) after derivatization with TMSI. Spectrum (a) was obtained with $100 \mathrm{ng}$ of adduct, whereas spectrum (b) was recorded with $5 \mathrm{ng}$ of TMSderivatized adduct. 
TMS groups reside on the carcinogen and/or base. A more completely derivatized pentakis-TMS product would result from attachment of two, rather than no TMS groups to the deoxyribose moiety. Bis modification of the deoxyribose has been reported for arylamine-deoxynucleoside adducts [17], and was sometimes observed in this study based on the observation of weak protonated molecules for pentakisTMS-derivatized products. For example, the deoxycytidine-BaPDE adduct gave an ion (spectrum not shown) that corresponds to a pentakis-TMS protonated molecule.

These results demonstrate the incomplete and sometimes variable TMS derivatization observed under the conditions used in this study. Attempts to use alternative derivatization strategies, including those already developed for arylamine-deoxynucleoside adducts, often resulted in decomposition of the PAH dihydrodiol-epoxide deoxynucleoside adducts. Nevertheless, except as noted below for the deoxyadenosine adduct of anti-BaPDE, an abundant tris-TMS-derivatized protonated molecule was observed for all of the TMS-modified PAH dihydrodiol-epoxide adducts. With the deoxyadenosine adduct of anti-BaPDE, neither a tris- nor a pentakis-TMS-protonated molecule was detected; only an ion that corresponds to the tris-TMS derivative of $\mathrm{BH}_{2}^{+}$was observed.

\section{Product Ions}

Underivatized adducts. To investigate the structural specificity of product ions from the adducts, and in particular, to determine if ions indicative of the site of base substitution could be identified, product ions were obtained from the protonated molecules of all the deoxyguanosine adducts. A FAB product ion spectrum of the protonated molecule $(\mathrm{m} / \mathrm{z} 546)$ for the antibenz[ $a$ ]anthracene dihydrodiol-epoxide deoxyguanosine adduct is shown in Figure 2. These results are in good agreement with data reported previously for FD

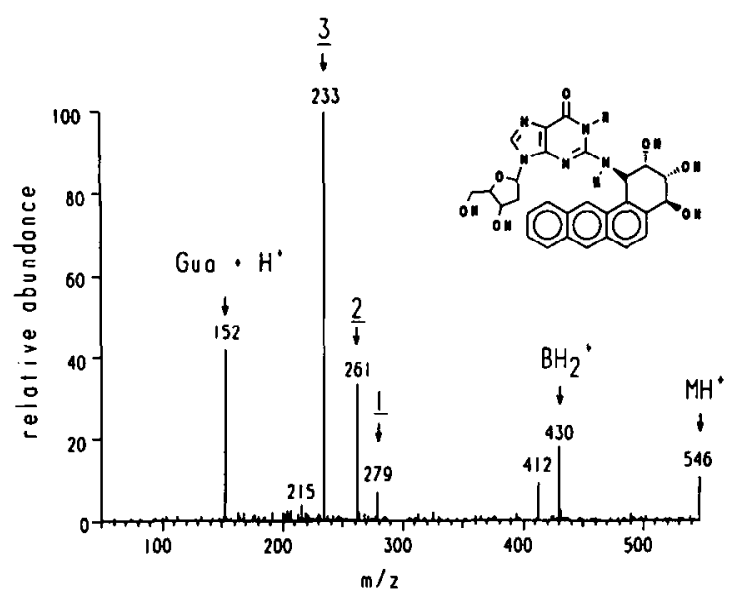

Figure 2. FAB product ion spectrum of the protonated molecule $(m / z 546)$ from $25 \mathrm{ng}$ of anti-benz $[a]$ anthracene dihydrodiolepoxide deoxyguanosine adduct.

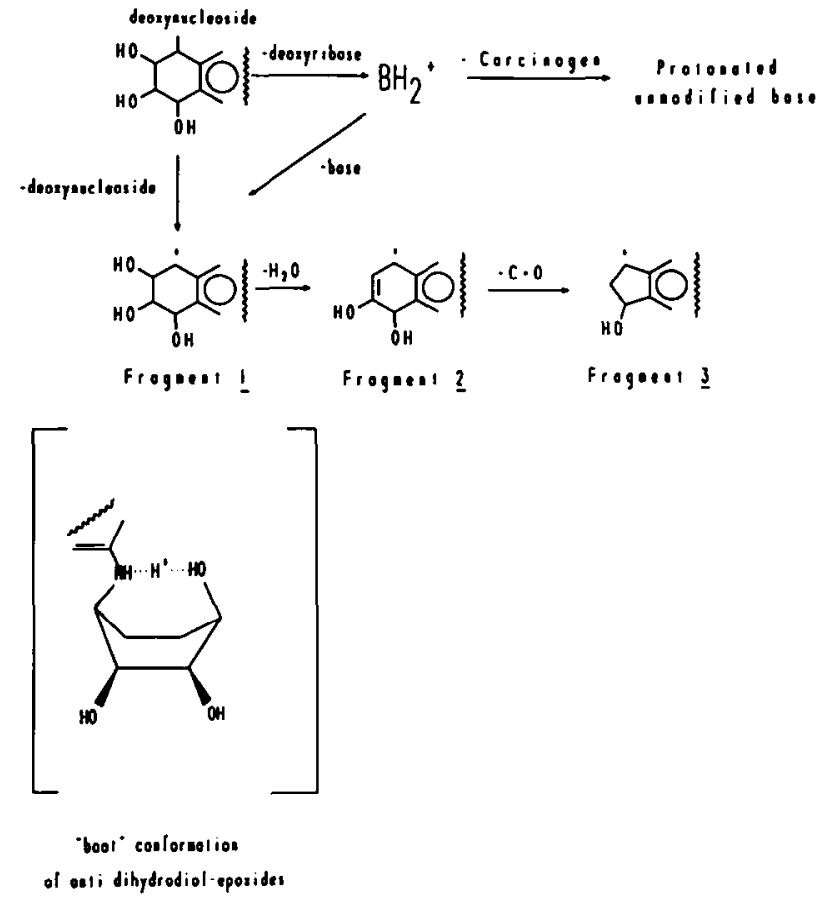

Scheme I. Proposed fragmentation pathway for the protonated molecules of PAH dihydrodiol-epoxide deoxynucleoside adducts. Initial cleavage of the $\mathrm{C}-\mathrm{N}$ bond between the base and carcinogen gives a carcinogen carbocation (fragment 1 ). Subsequent loss of 18 and $28 \mathrm{u}$ gives fragments 2 and 3, presumably due to loss of water and carbon monoxide, respectively. The latter fragment was typically the base peak. The "boat-like" conformation of the cyclohexane ring is shown below fragment 1 .

and CID mass spectrometry of BaPDE deoxynucleoside adducts $[10,11]$. Except for the greater abundance of $\mathrm{BH}_{2}^{+}$ion, the spectrum is typical of the results observed with the other PAH dihydrodiol-epoxide deoxyguanosine adducts. Fragment ions that correspond to loss of 267, 285, and $313 \mathrm{u}$ from the protonated molecule were common to all of the spectra, and the proposed structures for these fragments, labeled 1, 2, and 3, respectively, are shown in Scheme I. An ion that corresponds to the protonated, unmodified base (guanine, $m / z 152$ ) also was observed from each of the deoxyguanosine adducts. Similar results, which include fragments that correspond to 1,2 , and 3, were observed with deoxyguanosine-BaPDE, deoxyadenosine-BaPDE, and deoxycytidine-BaPDE adducts; however, the mass loss that gives rise to ion 1 is dependent on the mass of the leaving group (nucleobase). With these deoxynucleoside adducts, ions that correspond to the protonated bases were noted at $m / z 152$ (guanine), $m / z 136$ (adenine), and $m / z 112$ (cytosine).

Many of the product ions from the protonated molecule are attributed to initial cleavage of the sigma bond between the carbon atom of the PAH dihydrodiol-epoxide and the exocyclic amine ( $N^{2}$ for guanine) from the nucleobase. Formation of these ions can be explained based on the stability of carbocation structures $(1,2$, and 3$)$, initial cleavage of single rather than 
multiple bonds, and differences in the bond strengths for $\mathrm{C}-\mathrm{N}$ sigma bonds in aromatic amines as compared to aliphatic amines. This is accomplished most easily by using, as a frame of reference, the protonated molecule where the proton resides on the exocyclic nucleobase nitrogen. Migration of the pair of electrons that form the sigma bond between this nitrogen atom and the PAH carbon gives a stable carbocation that results from heterolytic cleavage of the $\mathrm{C}-\mathrm{N}$ bond. The absence of a significant signal for the ion that results from the alternative heterolytic $\mathrm{C}-\mathrm{N}$ cleavage between the nucleobase and the same exocyclic amine is consistent with the observation that for exocyclic amines, the $\mathrm{C}-\mathrm{N}$ bonds between an aromatic carbon (pyrimidine or purine) and an exocyclic amine are typically stronger than the same bond between a cyclic aliphatic carbon and an exocyclic amine (bond energies $\sim 4.4$ and $3.7 \mathrm{eV}$, respectively [20]). After the initial formation of fragment 1 , losses of water and then carbon monoxide result in the formation of two additional carbocations, fragments 2 and 3 , respectively.

The protonated base ion results from cleavage of the same $\mathrm{C}-\mathrm{N}$ bond that led to formation of ion 1 except that in this instance another hydrogen is transferred from the PAH to the amine nitrogen prior to cleavage. As a result the charge resides on the nucleobase side of the molecule. A proton transfer may be facilitated for the compounds investigated because of their conformation. The $\mathrm{OH}$ group on the opposite side of the cyclohexane ring is cis to the $\mathrm{NH}$ group and a "boatlike" conformation of the cyclohexane ring brings the $\mathrm{NH}$ and $\mathrm{OH}$ substituents on opposite sides of the cyclohexane ring into relatively close proximity (Scheme I). Such proximity and the resulting sharing of a proton was used to explain the relative stability (less fragmentation) of the cis isomer with respect to water loss in a study of protonated cis- and trans-1,4-cyclohexanediols [21]. In the current study the greater propensity for fragmentation, as opposed to less fragmentation from the cis-diols in ref 21, is attributed to the unequal sharing of a proton between nitrogen and oxygen. The proton affinity differences (typically about $1 \mathrm{eV}$ ) favor protonation of the nitrogen rather than the oxygen atom. Transfer of the charge-bearing proton (Scheme I) from oxygen to nitrogen would lead directly to fission of the PAH-nucleobase bond to give ion 1 (or the protonated base if the "extra" or chargebearing proton happened to reside elsewhere on the nucleoside base).

The absence of an analogous ion that contains the carcinogen moiety and the protonated amine can be attributed in part to the relative bond strengths cited previously, but additional factors such as the energy-entropy requirements for proton transfer from the nucleobase to the exocyclic amine likely play a role as well. The formation of ions 1,2 , and 3 could occur directly from the $\mathrm{MH}^{+}$ion or indirectly via the $\mathrm{BH}_{2}^{+}$ ion. The protonated base ion $(\mathrm{m} / z 152$ in Figure 2) does not contain a deoxyribose sugar and is thus attributed to fragmentation from an intermediate $\mathrm{BH}_{2}^{+}$ fragment.

Model compounds for the $\mathrm{BH}_{2}^{+}$ions were synthesized by reaction of anti-BaPDE with guanine, adenine, and cytosine (Figure 3). A representative product ion spectrum for the guanine-BaPDE adduct is shown in Figure 4 . The major fragments were the same as those observed from the protonated deoxynucleoside adducts, the structures of which were assigned in Scheme I.

RamaKrishna et al. [12] have conducted FAB product ion studies of BaPDE adducts of adenine and guanine with a four-sector mass spectrometer. The adducts, which were synthesized by electrochemical oxidation, were endocyclic N7-substituted products as compared to the exocyclic $N^{2}$ (guanine) and $N^{6}$ (adenine) substituted adducts shown in Figure 3. Although their spectra were acquired by using high energy conditions, the CID spectra were dominated by ions derived from the BaPDE moiety, with fragments 1,2 , and 3 (Scheme I) being readily apparent. These results suggest that product ion scans obtained from either low or high energy collisions provide structural information indicative of the molecular weights of the carcinogen and nucleobase, and perhaps some structural features. Unfortunately neither method provides fragment ions that are conclusive with regard to the site of carcinogen substitution.

Derivatized adducts. Figure 5 a shows a product ion scan $(m / z 570)$ of $25 \mathrm{ng}$ of the anti-BaPDE deoxyguanosine adduct, whereas Figure $5 \mathrm{~b}$ presents the product ion spectrum $(\mathrm{m} / \mathrm{z} 786)$ of $5 \mathrm{ng}$ of the same

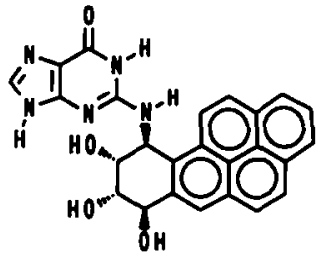

BaPDE-Guo

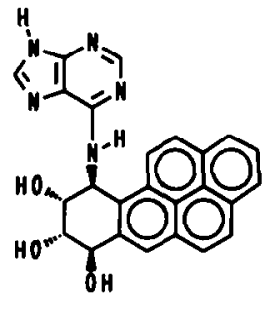

BoPDE - Ade

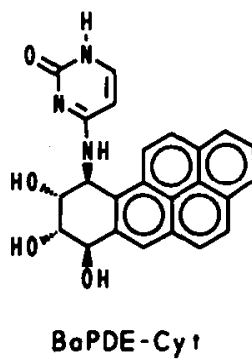

Figure 3. Structures of anti-BaPDE guanine adduct (BaPDEGua), anti-BaPDE adenine adduct (BaPDE-Ade), and anti-BaPDE cytosine adduct (BaPDE-Cyt). 


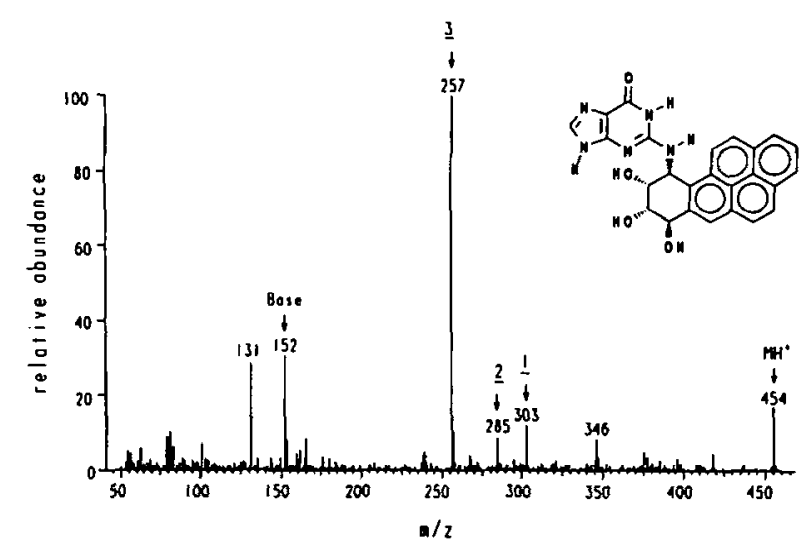

Figure 4. FAB product ion spectrum of the protonated molecule $(m / z 454)$ from anti-BaPDE guanine adduct.

adduct after derivatization with TMSI. These data are typical of the results observed with all of the TMS-derivatized deoxynucleoside adducts. Although greater sensitivity was obtained following derivatization, the product ion spectrum of the derivatized sample showed fewer fragments. For example, fragments that correspond to ions 1 and 3 in Scheme I (or TMS-derivatized analogs) were not observed with the derivatized adduct. A fragment that corresponds to bis-TMS-derivatized fragment 2 was present $(\mathrm{m} / z 429$ in Figure $5 \mathrm{~b}$ ); however, the most abundant fragment

a

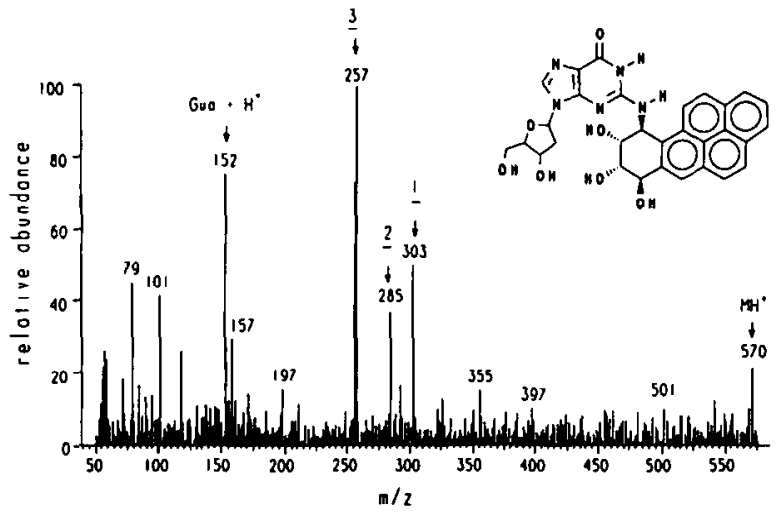

b

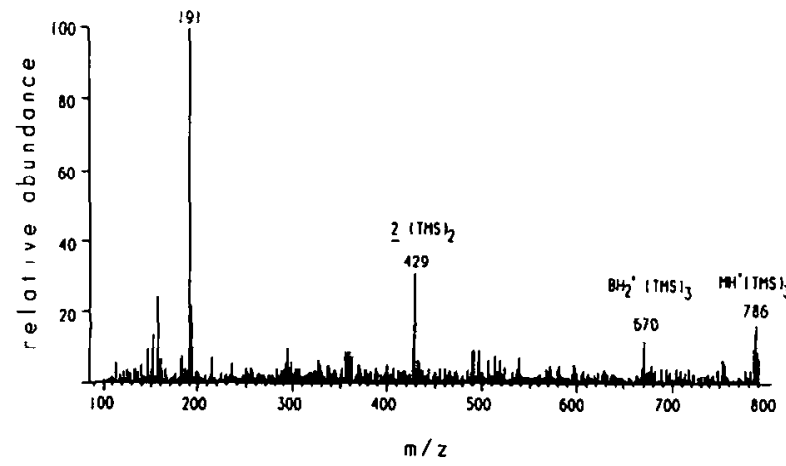

Figure 5. FAB product ion spectra from the protonated molecule of anti-BaPDE deoxyguanosine adduct (a) before $(\mathrm{m} / \mathrm{z} 570)$ and (b) after ( $m / z$ 786) derivatization with TMSI. Spectrum (a) was obtained with $25 \mathrm{ng}$ of adduct, whereas spectrum (b) was recorded with $5 \mathrm{ng}$ of TMS-derivatized adduct. ion is $m / z$ 191. A product ion scan (not shown) of this $m / z 191$ ion gave a base peak at $m / z 73$, which was presumed to be $\left(\mathrm{Si}\left(\mathrm{CH}_{3}\right)_{3}\right)^{+}$. This suggests that the ion of $m / z 191$ corresponds to $\left[\left(\left(\mathrm{CH}_{3}\right)_{3} \mathrm{SiO}\right)_{2} \mathrm{CH}\right]^{+}$. An ion at $m / z 191$ has been reported in the mass spectra of TMS derivatives of many polyhydroxy steroids; for example, a report by Brooks et al. [22] details a number of examples that show this and other rearrangements that involve the migration of TMS groups in mass spectra from trimethylsilyl ethers of steroids. Moreover, the structure of the $\mathrm{m} / \mathrm{z}$ 191 ion was reported by Gustafsson et al. to be $[\mathrm{TMSO}=\mathrm{CH}-\mathrm{OTMS}]^{+}[23]$.

With derivatized adducts, the $m / z 191$ fragment ion was also the base peak in product ion scans of the anti-BaPDE deoxyadenosine and deoxycytidine adducts. For the other dihydrodiol-epoxide deoxyguanosine adducts, the $m / z 191$ ion was prominent but the base peak was the protonated guanine base $(m / z$ 152).

\section{Constant Neutral Loss Scans}

Underivatized adducts. A characteristic loss to produce a $\mathrm{BH}_{2}^{+}$ion has been observed in mass spectra obtained from protonated nucleosides and nucleotides [24-26], carcinogen-modified nucleosides [17, 27, 28], and silylated carcinogen-modified nucleosides [15, 16]. By extending this observation, we demonstrated the utility of constant neutral loss scans (116 u) for the determination of nucleoside adducts of arylamines [17]. Constant neutral loss scans are particularly useful for analysis of unknown adducts because they can be acquired prior to product ion spectra to determine the molecular weight of the adduct.

Although many of the PAH dihydrodiol-epoxide adducts used in this study showed $\mathrm{BH}_{2}^{+}$ions as unimolecular (Figure 1a) or collision-induced (Figure 2) fragments, others did not show loss of the deoxyribose, even with collisional activation of the protonated molecule (Figure 5a). In all cases, the intensity of this ion was much less than was expected based on results previously reported for arylamine- and arylamidemodified deoxynucleosides [16, 17]. Nevertheless, constant neutral loss scans that correspond to loss of $116 \mathrm{u}$ were measured for all of the PAH dihydrodiol-epoxide adducts. For most of these adducts, loss of the deoxyribose from the protonated molecule, as well as the monosodium- and disodium-containing molecule ions, was observed (Figure 6a, for example). Another, more consistent type of constant neutral loss, based on the loss of $313 \mathrm{u}$, was observed for all of the deoxyguanosine-PAH dihydrodiol-epoxide adducts studied. This loss, which is attributed to consecutive losses of the components of water, carbon monoxide, and deoxyguanosine, gives rise to ion 3 in Scheme I. (The order of losses suggested in the scheme represents only one of several alternative mechanisms leading to an overall loss of 313 u.) Ion 3 is typically the base 

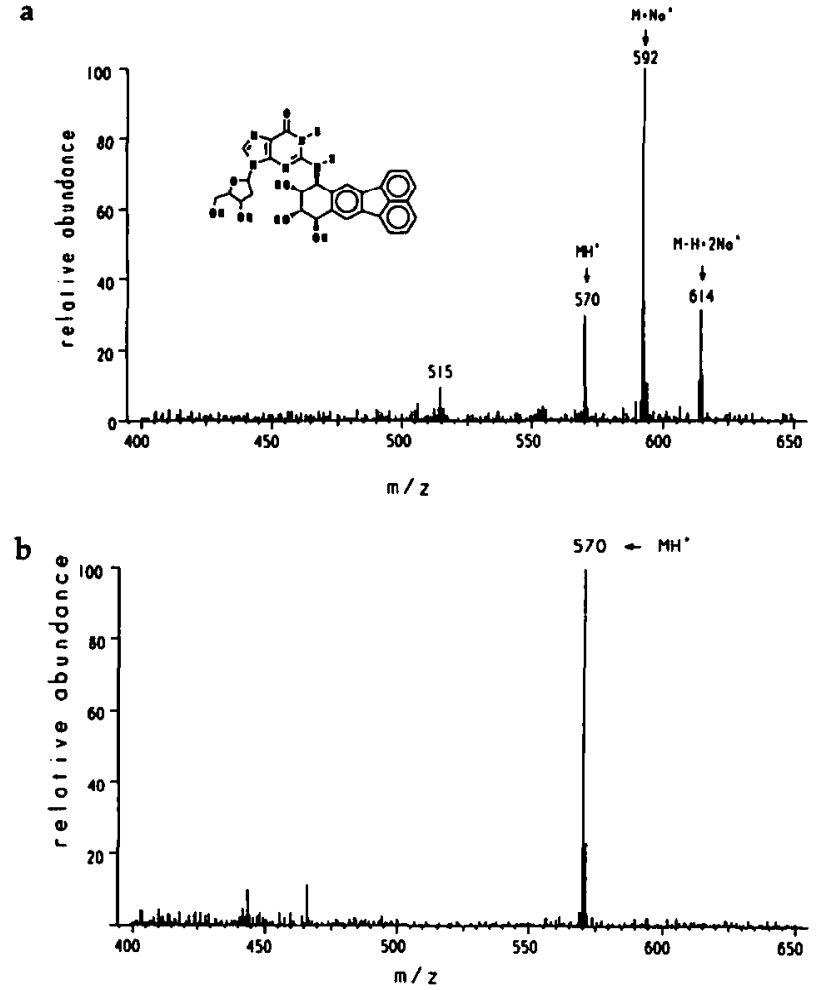

Figure 6. FAB constant neutral loss spectra of antibenzo[ $k$ ]fluoranthene dihydrodiol-epoxide deoxyguanosine adduct. Spectrum (a) follows the loss of $116 \mathrm{u}$; spectrum (b) follows the loss of $313 \mathrm{u}$. Similar results were obtained with the anti-benzo[ $b]$ fluoranthene dihydrodiol-epoxide deoxyguanosine adduct.

peak in product ion spectra of deoxyguanosine dihydrodiol-epoxide adducts. Consequently, when constant neutral loss scans were compared from identical samples, the loss of 313 u (Figure $6 \mathrm{~b}$ ) provided a more abundant signal than that observed with loss of $116 \mathrm{u}$ (Figure 6a).

Derivatized adducts. Unsatisfactory results were obtained with the constant neutral loss spectra of TMSderivatized PAH dihydrodiol-epoxide adducts. $\mathrm{Al}-$ though some adducts showed a loss of $116 \mathrm{u}$ in product ion spectra (e.g., Figure 5b), this loss was not observed universally in product ion or constant neutral loss spectra. This can be attributed to alternative fragmentation pathways to form ions that correspond to 2 (Scheme I) or $m / z$ 191. These results contrast with those previously obtained for TMS-derivatized arylamine adducts [17], where loss of $260 \mathrm{u}$ (bis-TMS derivatized deoxyribose) was observed for all of the arylamine adducts studied. In the present study, the hydroxyl groups unique to the dihydrodiol-epoxides were derivatized more readily (or the attached TMS groups were retained more readily) than those on the deoxyribose. Thus, constant neutral losses of 260 u can be measured only from protonated molecules of pentakis-TMS derivatives, and these were not always observed.

\section{Precursor Ion Scans}

Underivatized adducts. Product ion spectra for antiBaPDE adducts of deoxyguanosine, deoxyadenosine, and deoxycytidine showed a common base peak ion of $m / z 257$ (fragment 3 in Scheme I). These results suggested that if the identity of the PAH was known, precursor ion scans could be used to elucidate the identity of the deoxynucleoside that had been modified. Accordingly, when precursor ion spectra (257 u) were recorded for the three BaPDE adducts, protonated molecules were observed at $\mathrm{m} / \mathrm{z} 570$ (deoxyguanosine), 554 (deoxyadenosine), and 530 (deoxycytidine). A representative $\mathrm{FAB}$ precursor ion spectrum $(257 \mathrm{u})$ for the anti-BaPDE deoxyadenosine adduct is shown in Figure 7. The detection limit for this approach was typically around $10 \mathrm{ng}$.

Derivatized adducts. With underivatized adducts, precursor ion scans were based upon the PAH moiety (i.e., fragment 3 in Scheme I). TMS-derivatized adducts proved to be of greater utility for precursor ion scans because the $m / z 191$ ion was not dependent on either the PAH or the deoxynucleoside. (This ion, $m / z$ 191, has not been observed from TMS derivatives of other types of DNA adducts or from the TMS derivatization of biological and matrix blanks.) Thus, by using TMSderivatized adducts, information could be obtained on both the PAH dihydrodiol-epoxide and the deoxynucleoside. As an example, Figure 8 shows a precursor ion scan from DNA that had been reacted with antiBaPDE, enzymatically digested to deoxynucleosides, and then derivatized with TMSI. Two adducts were apparent: one was derived from deoxyguanosine, as evident from ions of $m / z$ 858, 786, and 670, which represent the tetrakis- and tris-TMS derivatives of the protonated molecule and the tris-TMS derivative of $\mathrm{BH}_{2}^{+}$, respectively. The other adduct resulted from deoxyadenosine, as indicated by the ion of $m / z 677$, which results from the tris-TMS $\mathrm{BHNa}^{+}$derivative.

To determine the utility of this technique for the analysis of mixtures that contain deoxynucleoside adducts from different PAH dihydrodiol-epoxides,

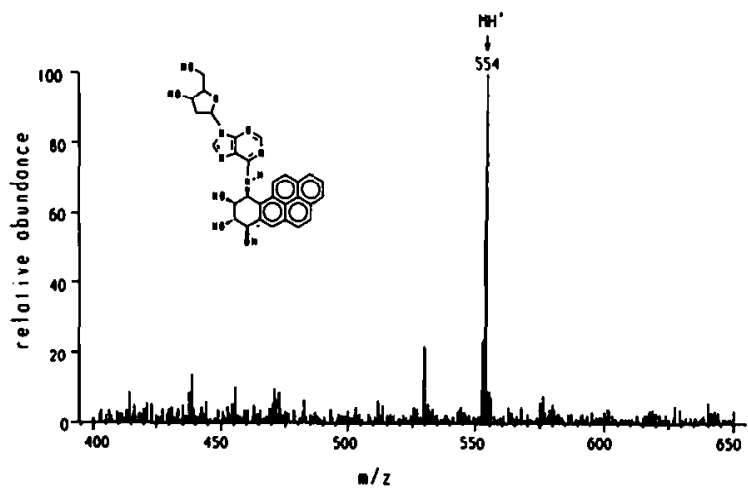

Figure 7. FAB precursor ion spectrum $(m / z 257)$ for $a n t i-B a P D E$ deoxyadenosine adduct. 


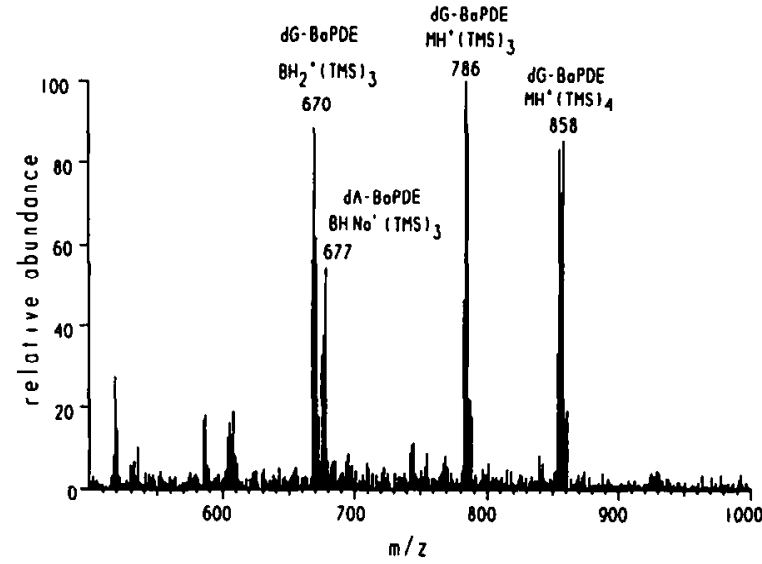

Figure 8. FAB precursor ion spectrum $(m / z 191)$ of DNA reacted with anti-BaPDE. Following enzymatic hydrolysis to deoxynucleosides, the hydrolysate was derivatized with TMSI.

DNA was reacted with anti-BaPDE, anti-chrysene dihydrodiol-epoxide, and anti-dibenz $[a, c]$-anthracene dihydrodiol-epoxide. The modified DNA was then hydrolyzed enzymatically to deoxynucleosides that were derivatized with TMSI. HPLC-UV analysis of the deoxynucleoside mixture before derivatization indicated the presence of the three deoxyguanosine adducts in a 4.2:5.8:1.0 ratio; however, only those adducts that resulted from reactions with anti-BaPDE and anti-chrysene dihydrodiol-epoxide were detected in the precursor ion scans (Figure 9). The ions of $m / z 670,786$, and 858 corresponded, respectively, to the tris-TMS derivative of $\mathrm{BH}_{2}^{+}$and the tris- and tetrakis-TMS derivatives of the protonated molecule of the anti-BaPDE deoxyguanosine adduct. Likewise, the ion of $\mathrm{m} / \mathrm{z} 646$ and perhaps the weaker signals for $m / z 762$ and 834 may be attributed to the tris-TMS derivative of $\mathrm{BH}_{2}^{+}$ and the tris- and tetrakis-TMS derivatives of the protonated molecule of the anti-chrysene dihydrodiolepoxide deoxyguanosine adduct. In addition, an antiBaPDE deoxyadenosine adduct was suggested by the

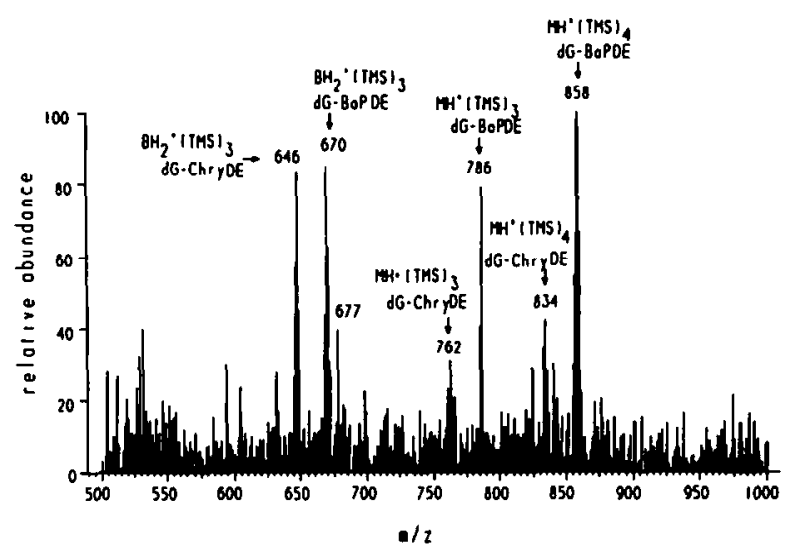

Figure 9. FAB precursor ion spectrum $(m / z 191)$ of DNA reacted with anti-BaPDE, anti-chrysene dihydrodiol-epoxide (ChryDE), and anti-dibenz $[a, c]$ anthracene dihydrodiol-epoxide. Following enzymatic hydrolysis to deoxynucleosides, the hydrolysate was derivatized with TMSI.

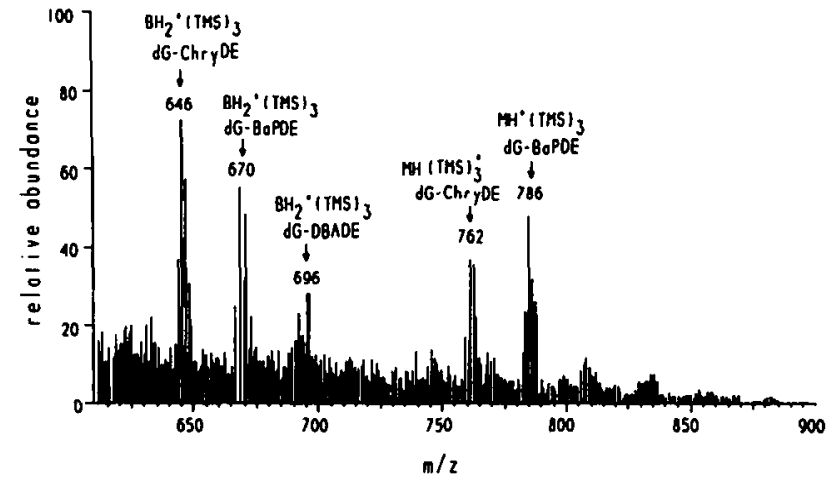

Figure 10. FAB mass spectrum of DNA reacted with antiBaPDE, anti-chrysene dihydrodiol-epoxide, and antidibenz $[a, c]$ anthracene dihydrodiol-epoxide (DBADE). Following enzymatic hydrolysis to deoxynucleosides, the hydrolysate was derivatized with TMSI.

presence of a weak ion of $m / z$ 677, which corresponded to the tris-TMS $\mathrm{BHNa}^{+}$derivative.

Constant neutral loss scans were obtained on the same mixture and only adducts obtained from antiBaPDE and anti-chrysene dihydrodiol-epoxide could be identified (spectra not shown); however, a FAB full scan spectrum (Figure 10) showed a small fragment ion of $\mathrm{m} / \mathrm{z} 696$ that corresponded to the tris-TMS derivative of the $\mathrm{BH}_{2}^{+}$of the deoxyguanosine dibenz $[a, c]$-anthracene dihydrodiol-epoxide adduct. The failure to detect this adduct in precursor ion or constant neutral loss scans is probably due to its low concentration relative to the other two deoxyguanosine adducts in the DNA sample.

\section{Conclusions}

These studies used three different experimental techniques (product ion, constant neutral loss, and precursor ion scans) to investigate $\mathrm{PAH}$ dihydrodiol-epoxide deoxynucleoside adducts. The results indicate that FAB tandem mass spectrometry has significant potential for the detection and structural characterization of PAH-dihydrodiol-epoxide as well as arylamine-DNA adducts, and the most appropriate technique depends on the specific adduct (e.g., class of carcinogen) and the information desired from the experiment. With a DNA adduct that results from a dihydrodiol-epoxide of an unknown $\mathrm{PAH}$, for example, constant neutral loss scans based upon loss of $313 \mathrm{u}$ (for deoxyguanosine adducts) proved to be a very sensitive method for PAH identification. Although this method may be applicable only to PAH dihydrodiol-epoxide adducts of deoxyguanosine, this particular deoxynucleoside is typically the major site for adduct formation in DNA [2].

In instances where the identity of the PAH dihydrodiol-epoxide is known, a precursor ion scan based upon a PAH fragment (i.e., fragment 3 in Scheme I) can be used to identify the molecular weight of the 
adduct and, hence, the deoxynucleoside. Alternatively, the mass of PAH dihydrodiol-epoxide initially can be determined by using constant neutral loss scans ( 313 or $116 \mathrm{u}$ ) and this can be followed by precursor ion scans to identify the deoxynucleoside. For adducts that result from carcinogens other than PAH dihydrodiolepoxides, constant neutral loss scans of $116 \mathrm{u}$, which represents the loss of deoxyribose, can be used to determine the molecular weight of the adduct, and then product ion scans can readily distinguish arylamine from PAH dihydrodiol-epoxide adducts due to abundant losses (e.g., $313 \mathrm{u}$ for the deoxy-guanosine$\mathrm{PAH}$ adduct) from the dihydrodiol-epoxide adducts.

Because of the more extensive fragmentation observed from the protonated molecules of PAH dihydrodiol-epoxide adducts, it is not necessary to conduct product ion studies using $\mathrm{BH}_{2}^{+}$ions, as was the case with the arylamine adducts previously studied [17]. Preparation of TMS derivatives increased the sensitivity of adduct detection. Precursor ion scans of these TMS derivatives, by using a 191-u fragment, allowed simultaneous detection of several DNA adducts in a mixture. These results suggest potential applications of tandem mass spectrometry techniques to the identification of PAH dihydrodiol-epoxide derived carcinogen-DNA adducts.

\section{Acknowledgments}

This work was supported in part by a grant from the Center for Indoor Air Research. We also thank one of the reviewers for insight regarding previous studies of rearrangements that involve TMS derivatives of diols and related compounds.

\section{References}

1. I.A.R.C. Monographs on the Evaluation of the Carcinogenic Risk of Chemicals to Humans; World Health Organization: Lyon, France, 1983; Vol. 32, pp 1-477.

2. Baird, W. M.; Pruess-Schwartz, D. In Polycyclic Aromatic Hydrocarbon Carcinogenesis: Structures-Activity Relationships, Vol. II; Shen, K.; Yang, B.; Silverman, D., Eds.; CRC Press: Boca Raton, FL, 1988; pp 141179.

3. Perera, F. Environ. Health Perspect. 1987, 76, 141.

4. Beland, F. A.; Poirier, M. C. In The Pathobiology of Neoplasin; Sirica, A. E., Ed.; Plenum: New York, 1989; pp 57-80.
5. Harris, C. C.; Vahakangas, K.; Newman, M.; Trivers, G. E.; Shamsuddin, A.; Sinopoli, N.; Mann, D. L.; Wright, W. C. Proc. Natl. Acad. Sci. USA 1985, 82, 6672.

6. Vahakangas, K.; Haugen, A.; Harris, C. Carcinogenesis 1985 , 6, 1109.

7. Gupta, R. C.; Reddy, M. V.; Randerath, K. Carcinogenesis 1982, 3, 1081.

8. Chiarelli, M. P.; Lay, J. O., Jr. Mass Spectrom. Rev. 1992, 11, 447.

9. Straub, K. M.; Meehan, T.; Burlingame, M. C. Proc. Natl. Acad. Sci. USA 1977, 74, 5285.

10. Straub, K. M.; Burlingame, A. L. Adr. Mass Spectrom. 1980, $8 B, 1127$.

11. Straub, K. M.; Burlingame, A. L.; Biomed. Mass Spectrom. $1981,8,431$.

12. RamaKrishna, N. V. S.; Gao, F.; Padmavathi, N. S.; Cavalieri, E. L.; Rogan, E. G.; Cerny, R. L.; Gross, M. L. Chem. Res. Toxicol. 1992, 5, 293.

13. Wiebers, J. L.; Abbott, P. J.; Coombs, M. M.; Livingston, D. C. Carcinogenesis 1981, 7,637.

14. Dino, J. J.; Guenat, C. R.; Tomer, K. B.; Kaufman, D. G. Rapid Commun. Mass Spectrom. 1987, 1, 69.

15. Silk, N. A.; Lay, J. O., Jr.; Martin, C. N. Biochem. Pharmacol. 1989, 38, 279.

16. Annan, R. S.; Giese, R. W.; Vouros, P. Anal. Biochem. 1990, 191, 86.

17. Bryant, M. S.; Chiarelli, M. P.; Lay, J. O., Jr. I. Am. Soc. Mass Spectrom. 1992, 3, 360.

18. Pulkrabek, P.; Leffler, S.; Weinstein, B.; Grunberger, D. Bioclemistry 1977, 16, 3127.

19. Heflich, R. H.; Morris, S. M.; Beranek, D. T.; McGarrity, L. J.; Chen, J. J.; Beland, F. A. Mutngenesis 1986, 1, 201.

20. McLafferty, F. W.; Turecek, F. Interpretation of Mass Spectra, 4th ed.; University Science Books: Mill Valley, CA, 1993; pp 343-344.

21. Winkler, J.; McLafferty, F. W. Tetrahedron 1974, 30, 2971.

22. Brooks, C. J. W.; Harvey, D. J.; Midleditch, B. S.; Vouros, P. Org. Mass Spectrom. 1973, 7, 925.

23. Gustafsson, J.-Å.; Ryhage, R.; Sjövall, J.; Moriarty, R. M. I. Amer. Chem. Soc. 1968, 91, 1234.

24. Schram, K. H. Trends Anal. Chem. 1988, 7, 28.

25. Moser, H.; Wood, G. W. Biomed. Envirom. Mass Spectrom. 1988, 15, 547.

26. Crow, F. W.; Tomer, K. B.; Gross, M. L.; McCloskey, J. A.; Bergstrom, D. E. Anal. Biochem. 1984, 139, 243.

27. Mitchum, R. K.; Evans, F. E.; Freeman, J. P.; Roach, D. Int. I. Mass Spectrom. Ion Phys. 1983, 46, 383.

28. Fu, P. P.; Miller, D. W.; Von Tungeln, L. S.; Bryant, M. S.; Lay, J. O., Jr.; Huang, K.; Jones, L.; Evans, F. E. Carcinogenesis 1991, 12, 609 . 